\title{
Controle químico da cigarrinha-do-milho e incidência dos enfezamentos causados por molicutes
}

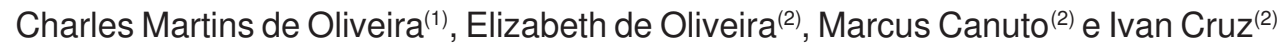

\begin{abstract}
(1)Embrapa Cerrados, Rod. BR 020, Km 18, Caixa Postal 08223, CEP 73310-970 Planaltina, DF. E-mail: charles@cpac.embrapa.br (2)Embrapa Milho e Sorgo, Rod. MG 424, Km 65, Caixa Postal 151, CEP 35701-970 Sete Lagoas, MG. E-mail: beth@cnpms.embrapa.br, mcanuto@gmail.com, ivancruz@cnpms.embrapa.br
\end{abstract}

\begin{abstract}
Resumo - O objetivo deste trabalho foi verificar a eficiência do tratamento inseticida de sementes de milho no controle de Dalbulus maidis e na redução da incidência de enfezamentos em viveiro telado e em campo. Foram realizados dois experimentos; no experimento 1 , em viveiro telado, sementes de milho foram tratadas com imidacloprid e thiamethoxan e, nessas plantas, cigarrinhas sadias, cigarrinhas infectantes com fitoplasma ou com espiroplasma foram confinadas. Avaliaram-se eficiência de controle, incidência de plantas com enfezamentos, altura das plantas e produção de grãos. No experimento 2 , em campo, imidacloprid e thiamethoxan foram utilizados em tratamento de sementes e pulverizações aos 10 e 20 dias após a semeadura. A incidência de enfezamentos e a produção de grãos foram avaliadas. Em viveiro telado, os produtos imidacloprid e thiamethoxan proporcionaram controle de adultos de D. maidis acima de 50\%, até o trigésimo dia, e reduziram a incidência de doenças e danos no crescimento e produção das plantas infectadas expostas às cigarrinhas infectantes aos dois dias após a emergência. Em campo, não foi constatada redução na incidência de enfezamentos ou ganho em produção, possivelmente devido ao fluxo migratório de cigarrinhas infectantes.
\end{abstract}

Termos para indexação: Zea mays, Dalbulus maidis, Spiroplasma kunkelii, neonicotinóides, fitoplasma.

\section{Chemical control of corn leafhopper and incidence of corn stunting diseases caused by mollicutes}

\begin{abstract}
The objective of this work was to verify the efficiency of maize seed treatment on Dalbulus maidis control, and its effect on corn stunting diseases incidence, at screenhouse and at field. Two experiments were carried out. In the experiment 1 ( screenhouse), maize seeds were treated with imidacloprid or with thiamethoxan. Healthy leafhoppers, phytoplasma infective leafhoppers, and spiroplasma infective leafhoppers were confined in plants. Efficiency of control, incidence of plants with corn stunting diseases, plant height, and grain production were evaluated. In the experiment 2 (field) imidacloprid and thiamethoxan were used for seed treatment and sprayed at 10 and 20 days after sowing. Corn stunting diseases incidence and grain production were evaluated. At screenhouse, the use of imidacloprid and thiamethoxan controlled the adults of $D$. maidis up to $50 \%$, until the $30^{\text {th }}$ day, and reduced disease incidence and damage on growth and grain production of the infected plants exposed to the infective leafhoppers, two days after emergency. In field, redution on corn stunting diseases or increasing production due to seed treatments was not observed possibly because of the migratory flux of infective leaf hoppers.
\end{abstract}

Index terms: Zea mays, Dalbulus maidis, Spiroplasma kunkelii, neonicotinoids, phytoplasma.

\section{Introdução}

No manejo integrado de pragas (MIP), as decisões quanto às medidas de controle devem-se fundamentar em diversos fatores relacionadas à praga, à cultura e ao ambiente. Neste sentido, é importante determinar o nível do dano econômico (NDE), que possibilita a utilização dessas medidas de controle, buscando menores gastos e menor impacto ambiental.
Pragas-chave, que causam danos indiretos às culturas pela transmissão de fitopatógenos, por exemplo, impossibilitam a determinação do NDE já que os danos não são proporcionais ao tamanho da população, o que torna necessário o uso de medidas preventivas (Pedigo, 1999).

Dalbulus maidis (DeLong \& Wolcott, 1923) (Hemiptera: Cicadellidae) é uma espécie responsável por perdas expressivas na produção do milho, por transmitir 
para as plantas os molicutes, fitoplasma e espiroplasma (Spiroplasma kunkelii Whitcomb), agentes causais do enfezamento do milho, e também do Maize rayado fino marafivirus (MRFV). Prejuízos causados por essas doenças têm sido observados, principalmente, na região Centro-Oeste e em áreas onde se planta o milho de segunda época ou safrinha (Oliveira et al., 2002b).

Embora o uso de cultivares resistentes seja o método de controle mais eficiente e recomendado para o controle dessas doenças (Shurtleff, 1980; Power, 1987), nem todas as cultivares de milho disponíveis no mercado possuem resistência satisfatória e, por isso, alternativas para seu controle é altamente desejável. Alternativas para redução na população da cigarrinha visando a redução na incidência dessas doenças têm sido avaliadas (Power, 1989).

O controle químico de D. maidis foi testado por alguns autores com resultados inconsistentes (Bhirud \& Pitre, 1972; Perfecto, 1990; Tsai et al., 1990). A utilização de inseticidas para a proteção das plantas de milho, principalmente na fase inicial de seu desenvolvimento, quando ocorre a infecção pelos molicutes (Hruska \& Peralta, 1997; Massola Júnior et al., 1999), pode ser uma das alternativas para o manejo desta praga. No Brasil, apesar da possibilidade de controle de $D$. maidis em campos de sementes, como ressaltaram Oliveira et al. (1997), são raros os estudos sobre esse tema. Atualmente, os produtos imidacloprid e thiamethoxan, além de clotianidina, são os únicos registrados no Ministério da Agricultura, Pecuária e Abastecimento para controle da cigarrinha-do-milho.

Este trabalho teve como objetivos avaliar a eficiência do tratamento inseticida de sementes no controle da cigarrinha $D$. maidis e na redução da transmissão de molicutes por cigarrinhas infectantes assim como a eficácia do controle químico da cigarrinha na incidência de enfezamentos, sob condições de campo.

\section{Material e Métodos}

Foram realizados dois experimentos em Sete Lagoas, MG, na área experimental da Embrapa Milho e Sorgo: o primeiro, em viveiro telado com delineamento inteiramente ao acaso num esquema fatorial $3 \times 3 \times 5$, com dez repetições, num total de 450 plantas. $O$ primeiro fator, correspondeu a cigarrinhas D. maidis infectadas com fitoplasma, ou com espiroplasma ou isentas de fitopatógenos; o segundo fator, a sementes de milho tratadas com inseticidas imidacloprid, ou com thiamethoxan ou sementes não tratadas, e o terceiro fator, a idade em que as plantas foram submetidas à alimentação pelas cigarrinhas $(2,9,16,23$ e 30 dias após a emergência das plantas).

Sementes de milho-pipoca, variedade Pop Zélia, tratadas com imidacloprid, com thiamethoxan, e também sementes não tratadas, foram semeadas em potes de plástico $(5 \mathrm{~kg})$ com solo previamente analisado e adubado, sendo três sementes por vaso. Todas as plantas utilizadas no experimento foram plantadas no mesmo dia e, dois dias após a emergência, realizou-se o desbaste, e apenas uma planta permaneceu em cada vaso. Antes do início do estudo, grupos de ninfas sadias de $D$. maidis de terceiro ou quarto ínstar foram colocadas em plantasfonte de fitoplasma e espiroplasma mantidas em casa de vegetação. Foram preparados cinco grupos de ninfas, espaçados uns dos outros por intervalos de sete dias. As ninfas de cada grupo passaram pelo período de acesso à aquisição dos fitopatógenos (quatro dias) e pelo período latente (três semanas). Terminado esse período, os insetos, já adultos, estavam infectivos e aptos a transmitir os fitopatógenos.

Aos 2, 9, 16, 23 e 30 dias após a emergência das plantas, grupos de cinco adultos de cigarrinha D. maidis, infectantes com fitoplasma, ou infectantes com espiroplasma ou isentos de fitopatógenos, com idade aproximada de duas a três semanas, foram colocados, separadamente, para se alimentar em plantas tratadas com cada produto inseticida e na testemunha, utilizando-se dez repetições. Utilizou-se um lote diferente de plantas em cada data. As plantas foram cobertas com gaiolas de confinamento, constituídas de garrafas de plástico transparente (2 L), cujo fundo foi retirado. Essas garrafas possuíam aberturas laterais protegidas por tecido "voile" para ventilação, conforme Oliveira \& Lopes (2004). Os insetos foram introduzidos nas gaiolas, por meio de um aspirador bucal, pela abertura superior da garrafa, que posteriormente foi fechada com tampa de rosca.

A avaliação de mortalidade foi realizada 96 horas após o confinamento das cigarrinhas, em cada uma das datas (2, 9, 16, 23 e 30 dias após emergência das plantas). Foram considerados mortos apenas os insetos que não apresentavam movimentos dos apêndices locomotores (pernas e asas). Após o período de inoculação de 96 horas, as plantas submetidas às cigarrinhas foram mantidas em viveiro telado. Foram realizadas adubações com sulfato de amônio $\left(0,25 \mathrm{~g} \mathrm{~kg}^{-1}\right)$, semanalmente, até o florescimento. 
As plantas foram avaliadas quanto à presença de sintomas foliares de enfezamentos aos 100 dias após plantio. Foram considerados sintomas de infecção por fitoplasma ou espiroplasma, a presença de avermelhamento ou amarelecimento nas margens ou no ápice das folhas, presença de estrias esbranquiçadas, iniciando-se na base das folhas, e também a seca foliar precoce. As plantas foram conduzidas até a produção e determinada a altura de plantas e o peso total de grãos por planta.

O segundo experimento foi conduzido em campo. Utilizou-se a cultivar BR 3123, por ser um híbrido susceptível aos enfezamentos do milho, o que facilita a avaliação de incidência por meio dos sintomas foliares. Utilizou-se o tratamento de sementes com os inseticidas thiamethoxan e imidacloprid associados ou não com pulverizações desses mesmos inseticidas em diferentes datas, em um total de 15 tratamentos: testemunha (sementes não tratadas - sem pulverização); sementes não tratadas - pulverização de thiamethoxan aos 10 dias após a emergência das plantas (DAE); sementes não tratadas - pulverizações de thiamethoxan aos $10 \mathrm{e}$ 20 DAE; sementes não tratadas - pulverização de imidacloprid aos $10 \mathrm{DAE}$; sementes não tratadas pulverizações de imidacloprid aos 10 e 20 DAE; sementes tratadas com imidacloprid - sem pulverização; sementes tratadas com imidacloprid - pulverização de thiamethoxan aos 10 DAE; sementes tratadas com imidacloprid - pulverizações de thiamethoxan aos $10 \mathrm{e}$ 20 DAE; sementes tratadas com imidacloprid pulverização de imidacloprid aos $10 \mathrm{DAE}$; sementes tratadas com imidacloprid - pulverizações de imidacloprid aos 10 e 20 DAE; sementes tratadas com thiamethoxan - sem pulverização; sementes tratadas com thiamethoxan - pulverização de thiamethoxan aos 10 DAE; sementes tratadas com thiamethoxan pulverizações de thiamethoxan aos 10 e 20 DAE; sementes tratadas com thiamethoxan - pulverização de imidacloprid aos $10 \mathrm{DAE}$; sementes tratadas com thiamethoxan - pulverizações de imidacloprid aos $10 \mathrm{e}$ 20 DAE. O delineamento experimental foi o de blocos ao acaso, com quatro repetições. Cada parcela experimental foi composta de dez fileiras de $7 \mathrm{~m}$ de comprimento, com espaçamento de $0,9 \mathrm{~m}$ entre fileira e $0,2 \mathrm{~m}$ entre plantas, sendo a área útil constituída pelas oito fileiras centrais, com distância de $1 \mathrm{~m}$ entre parcelas.

Sementes de milho foram tratadas com imidacloprid (480 g por $100 \mathrm{~kg}$ de sementes) ou com thiamethoxan (200 g por $100 \mathrm{~kg}$ de sementes) ou não foram tratadas.
Aos 10 e 20 dias após a emergência das plantas, de acordo com cada tratamento, foi realizada a pulverização com os mesmos inseticidas (imidacloprid $175 \mathrm{~g} \mathrm{ha}^{-1} \mathrm{e}$ thiamethoxan $37,5 \mathrm{~g} \mathrm{ha}^{-1}$ ), utilizando-se um pulverizador costal com bico 8004 e vazão de $250 \mathrm{~L} \mathrm{ha}^{-1}$, tendo-se o cuidado de cercar a parcela a ser pulverizada com uma lona de plástico para evitar que a deriva dos produtos atingisse parcelas que não receberiam a pulverização. $\mathrm{O}$ experimento foi conduzido observando-se todos os tratos culturais recomendados para a cultura do milho na região. A infestação da cultura por populações do inseto vetor $D$. maidis foi natural.

Havia, ao lado da área experimental, por ocasião da instalação do estudo, um campo de milho em fase de enchimento de grãos, com plantas apresentando sintomas típicos de enfezamentos, que poderia servir como fonte de populações de cigarrinhas portadoras dos molicutes.

Para a avaliação da eficiência dos tratamentos, aos 100 dias após a emergência, foi avaliada a incidência de enfezamentos. Todas as plantas da área útil de cada parcela foram avaliadas quanto à presença de sintomas foliares típicos de enfezamentos, conforme descrito no experimento 1 . Posteriormente as plantas foram conduzidas até o final do ciclo e avaliada a produção e o número de espigas por planta.

No experimento 1 , os dados de mortalidade dos tratamentos (\%) foram utilizados para o cálculo da eficiência dos inseticidas por meio da fórmula de Abbott (1925) e, assim como os dados de porcentagem de sintomas foliares, avaliados nos experimentos 1 e 2 , foram transformados segundo $\operatorname{arc} \operatorname{sen}(x / n)^{0,5}$, e no caso de $x=0$ e $x=n$ utilizouse $\operatorname{arcsen}(1 / 4 n)^{0,5}$ e $\operatorname{arcsen}(4 n-1 / 4 n)^{0,5}$, respectivamente (Pimentel-Gomes, 2000). Os dados de altura de plantas, do experimento 1, e de produção, dos experimentos 1 e 2 , não foram transformados. Os dados dos dois experimentos foram submetidos a análises de variância e as médias comparadas pelo teste de Scott-Knott, a 5\% de probabilidade, utilizando-se o SISVAR 4.3 (Ferreira, 2000).

\section{Resultados e Discussão}

No experimento em viveiro telado detectou-se interação significativa entre os fatores datas de avaliação e inseticidas, quanto à eficiência de controle das cigarrinhas (Tabela 1). Nas variáveis sintoma, altura de planta e peso seco de grãos, a interação foi significativa entre todos os fatores testados (Tabela 2). Independentemente da infectividade das cigarrinhas - 
sadias ou infectantes com molicutes -, imidacloprid e thiamethoxan apresentaram alta eficiência de controle até o $23^{\circ}$ dia $(\geq 70 \%)$. Esta porcentagem foi semelhante até o $16^{\circ}$ dia, para o primeiro, e até o 9o dia para o segundo produto, respectivamente. Os dois inseticidas apresentaram porcentagens de eficiência de controle da cigarrinha-do-milho semelhantes em todas as datas de inoculação, com exceção da inoculação realizada aos 16 dias após a emergência das plantas em que imidacloprid mostrou-se significativamente superior ao thiamethoxan (Tabela 1).

Em estudos com o pulgão Myzus persicae, na cultura da alface, os dois inseticidas apresentaram alta eficiência de controle até os 43 dias após o transplante (Zagonel et al., 2002). Na cultura da batata, a utilização de imidacloprid, aplicado no solo, mostrou alta eficiência de controle nas espécies de pulgão Macrosiphum euphorbiae, M. persicae e Aphis nasturtii, por no mínimo 62-65 dias após a emergência. Contudo, a aplicação foliar do produto requereu no mínimo cinco dias de alimentação dessas espécies para uma porcentagem de controle de 50-75\% (Boiteau et al., 1997). Nos percevejos-da-soja, Piezodorus guildinii, Nezara viridula e Euschistus heros, uma mistura de thiamethoxan, cipermetrina e sal de cozinha permitiu o controle dessas espécies por até 15 dias (Ramiro et al., 2005). A eficiência de controle dos inseticidas thiamethoxan e imidacloprid, de acordo com seu efeito residual, é variável em diferentes espécies-praga e com a dose do produto (Boiteau et al., 1997). Apenas plantas submetidas a cigarrinhas infectantes apresentaram sintomas de enfezamentos (Tabela 2).

$\mathrm{O}$ tratamento inseticida de sementes, para ambos produtos, causou redução de forma variável na incidência de plantas com sintomas de enfezamentos, sendo esse efeito significativamente maior quando a inoculação foi feita aos dois dias após a emergência das plântulas, e marcante para imidacloprid. Esse efeito persistiu até a inoculação aos nove dias após a emergência das plântulas, quando as sementes foram tratadas com imidacloprid. Há relatos de que as cigarrinhas necessitam alimentar-se cerca de 0,5 e 1 hora para transmissão do fitoplasma e do espiroplasma, respectivamente (Markham \& Alivizatos, 1983; Legrand \& Power, 1994), e, nesse caso, podem ter morrido antes de completar a transmissão. A redução na incidência de plantas com sintomas de enfezamentos pode ser atribuída à alta e rápida mortalidade das cigarrinhas proporcionadas pelos inseticidas (Tabela 1), sugerindo que o tratamento inseticida de sementes de milho, com esses produtos poderia reduzir o inóculo inicial dos molicutes, matando as cigarrinhas infectantes que chegam à lavoura nos estádios iniciais de desenvolvimento das plantas (Oliveira et al., 2002a). Os enfezamentos são doenças consideradas de ciclo primário, cujo inóculo não se multiplica dentro da lavoura, em virtude do desenvolvimento relativamente lento dos molicutes nas plantas de milho, que podem necessitar cerca de 30 dias para serem detectados na parte aérea (Oliveira et al., 2002c). Já foi demostrado que o espiroplasma, ao infectar plântulas de milho, movimenta-se primeiro em direção às raízes, e depois em direção à parte aérea (Gussie et al., 1995). D. maidis tem hábito migratório e dissemina os molicutes de lavouras de milho doentes, para plântulas jovens em lavouras recém-estabelecidas (Oliveira et al., 2002a). É possível supor que a redução na porcentagem de cigarrinhas infectantes que chegam em uma lavoura de milho recém-germinada, por meio do tratamento de sementes, poderia reduzir a incidência de plantas com enfezamentos.

A redução na incidência de plantas com sintoma de enfezamento, verificada quando as plantas foram expostas às cigarrinhas infectantes aos 30 dias após a germinação, pode ser atribuída à interferência de outros fatores como a expressão de resistência pelas plantas, atuando em sinergismo, e de forma diferenciada, com

Tabela 1. Eficiência média (\% de mortalidade) dos inseticidas utilizados no tratamento de sementes no controle de adultos de Dalbulus maidis em função da idade das plantas ${ }^{(1)}$.

\begin{tabular}{|c|c|c|c|c|c|c|c|c|}
\hline \multirow[t]{2}{*}{$\operatorname{Idade}^{(2)}$} & \multicolumn{4}{|c|}{ Imidacloprid } & \multicolumn{4}{|c|}{ Thiamethoxan } \\
\hline & Fitoplasma ${ }^{(3)}$ & Espiroplasma $^{(4)}$ & Sadia $^{(5)}$ & Média & Fitoplasma & Espiroplasma & Sadia & Média \\
\hline 2 & 100 & 100 & 96 & $99 \mathrm{aA}$ & 100 & 98 & 100 & $99 \mathrm{aA}$ \\
\hline 9 & 100 & 100 & 100 & $100 \mathrm{aA}$ & 98 & 100 & 95 & $98 \mathrm{aA}$ \\
\hline 16 & 97 & 100 & 93 & $97 \mathrm{aA}$ & 84 & 88 & 85 & $86 b B$ \\
\hline 23 & 81 & 79 & 78 & $79 \mathrm{aB}$ & 75 & 70 & 71 & $72 \mathrm{aC}$ \\
\hline 30 & 52 & 51 & 60 & $54 \mathrm{aC}$ & 59 & 55 & 53 & $56 \mathrm{aD}$ \\
\hline
\end{tabular}

${ }^{(1)}$ Médias seguidas de mesma letra, minúscula nas linhas e maiúscula nas colunas, não diferem entre si pelo teste de Scott-Knott, a 5\% de probabilidade. ${ }^{(2)}$ Idade das plantas (dias após a emergência) no momento em que foram submetidas às cigarrinhas. ${ }^{(3)}$ Cigarrinhas adultas de $D$. maidis infectantes com fitoplasma. ${ }^{(4)}$ Cigarrinhas adultas de D. maidis infectantes com espiroplasma. ${ }^{(5)}$ Cigarrinhas adultas de D. maidis sadias. 
os produtos inseticidas e ao comportamento diferenciado dos dois fitopatógenos com relação à capacidade de infecção dos tecidos da planta. Foi demonstrado que genótipos de milho diferentes apresentam resistência diferenciada ao fitoplasma e ao espiroplasma (Silva et al., 2002, 2003; Oliveira et al., 2005).

$\mathrm{O}$ aumento na incidência de plantas com sintomas, de acordo com o aumento da idade das plantas, entre 2 e 30 dias após a emergência, pode ser decorrente da diluição dos inseticidas na seiva com conseqüente aumento do tempo de alimentação necessário para causar a morte das cigarrinhas, o que permitiu a transmissão dos molicutes para as plantas. Broadbent (1957) relatou que a atividade de inseticidas sistêmicos varia com a idade das plantas. Esses inseticidas diminuem seu efeito à medida que as plantas crescem. Tem sido demonstrado experimentalmente que o uso de inseticidas é capaz de controlar a população de insetos vetores por períodos relativamente longos, sem que, no entanto, haja redução significativa das doenças por eles disseminadas (Broadbent, 1957; Boiteau et al., 1997). A ação lenta dos inseticidas para levar à morte os insetos vetores, conforme relatado para imidacloprid, pode ser uma das causas da ineficiência na prevenção de disseminação de doenças em plantas (Boiteau et al., 1997).
Reduções significativas na altura das plantas foram observadas nos tratamentos de inoculação aos 9 e aos 16 dias após a emergência das plantas para o imidacloprid (Tabela 2). Esse efeito foi maior quando as plantas foram tratadas com thiamethoxan $(2,9,16 \mathrm{e}$ 23 dias), o que pode ser evidenciado pela maior incidência de plantas com sintomas de enfezamentos nesse tratamento (Tabela 2). Essa redução foi significativamente maior em tratamentos de inoculação com espiroplasma, em relação aos tratamentos de inoculação com fitoplasma, confirmando a diferença, anteriormente relatada, no efeito de fitoplasma e de espiroplasma sobre o desenvolvimento de plantas de milho (Oliveira et al., 2002c).

Com relação à severidade dos danos causados por esses fitopatógenos na produtividade de grãos, em geral, os maiores efeitos negativos, para os dois produtos, foram observados quando as plantas foram submetidas às cigarrinhas infectantes aos 9, 16 e 23 dias após a emergência. As reduções na produtividade foram menores quando a inoculação foi realizada aos dois dias após a emergência, quando o uso dos inseticidas reduziu significativamente a infecção pelos molicutes; e quando as plantas foram infectadas tardiamente aos 30 dias após a emergência. Tem sido demonstrado que infecções tardias das plantas de

Tabela 2. Porcentagem média de plantas que apresentaram sintomas foliares de enfezamento (100 dias após emergência), altura média (m) e produção média de grãos (g por planta) de plantas de milho-pipoca submetidas ou não ao tratamento inseticida de sementes e à cigarrinhas, em viveiro telado ${ }^{(1)}$.

\begin{tabular}{|c|c|c|c|c|c|c|c|c|c|}
\hline \multirow[t]{2}{*}{$\operatorname{Idade}^{(2)}$} & \multicolumn{3}{|c|}{ Imidacloprid } & \multicolumn{3}{|c|}{ Thiamethoxan } & \multicolumn{3}{|c|}{ Semente não tratada } \\
\hline & Fitoplasma $^{(3)}$ & Espiroplasma $^{(4)}$ & Sadia $^{(5)}$ & Fitoplasma & Espiroplasma & Sadia & Fitoplasma & Espiroplasma & Sadia \\
\hline & \multicolumn{9}{|c|}{ Porcentagem de plantas com sintomas } \\
\hline 2 & $10 \mathrm{aA} A$ & $10 \mathrm{aA} A$ & $0 \mathrm{aA} A$ & $30 \mathrm{bB} A$ & $60 \mathrm{cA} B$ & $0 \mathrm{aA} A$ & $100 \mathrm{bB} B$ & $100 \mathrm{bAC}$ & $0 \mathrm{aA} A$ \\
\hline 9 & $60 \mathrm{bB} A$ & $70 \mathrm{bB} A$ & $0 \mathrm{aA} A$ & $100 \mathrm{bC} B$ & $100 \mathrm{bB} B$ & $0 \mathrm{aA} A$ & $100 \mathrm{bB} B$ & $100 \mathrm{bA} B$ & $0 \mathrm{aA} A$ \\
\hline 16 & $100 \mathrm{bC} A$ & $90 \mathrm{bC} A$ & $0 \mathrm{aA} A$ & $100 \mathrm{bC} A$ & $100 \mathrm{bB} A$ & $0 \mathrm{aA} A$ & $100 \mathrm{bB} A$ & $100 \mathrm{bA} A$ & $0 \mathrm{aA} A$ \\
\hline 23 & $70 \mathrm{bB} A$ & $100 \mathrm{cC} A$ & $0 \mathrm{aA} A$ & $80 \mathrm{bC} A$ & $100 \mathrm{bB} A$ & $0 \mathrm{aA} A$ & $80 \mathrm{bB} A$ & $100 \mathrm{bA} A$ & $0 \mathrm{aA} A$ \\
\hline \multirow[t]{2}{*}{30} & $10 \mathrm{aA} A$ & $70 \mathrm{bB} A$ & $0 \mathrm{aA} A$ & $0 \mathrm{aA} A$ & $100 \mathrm{bB} A$ & $0 \mathrm{aA} A$ & $0 \mathrm{aA} A$ & $80 \mathrm{bA} A$ & $0 \mathrm{aA} A$ \\
\hline & \multicolumn{9}{|c|}{ Altura de plantas } \\
\hline 2 & $1,52 \mathrm{aB} A$ & $1,54 \mathrm{aA} A$ & $1,66 \mathrm{aA} A$ & $1,57 \mathrm{aB} A$ & $1,04 \mathrm{bC} B$ & $1,64 \mathrm{aA} A$ & $1,22 \mathrm{bC} B$ & $0,59 \mathrm{cDC}$ & $1,74 \mathrm{aA} A$ \\
\hline 9 & $1,66 \mathrm{aB} A$ & $1,25 \mathrm{bB} A$ & $1,74 \mathrm{aA} A$ & $1,38 \mathrm{bC} B$ & $0,75 \mathrm{cD} B$ & $1,74 \mathrm{aA} A$ & $1,25 \mathrm{bC} B$ & $0,64 \mathrm{cD} B$ & $1,59 \mathrm{aA} A$ \\
\hline 16 & $1,69 \mathrm{aB} A$ & $1,35 \mathrm{bB} A$ & $1,89 \mathrm{aA} A$ & $1,59 \mathrm{bB} A$ & $1,09 \mathrm{cC} B$ & $1,86 \mathrm{aA} A$ & $1,68 \mathrm{aB} A$ & $1,18 \mathrm{bC} B$ & $1,76 \mathrm{aA} A$ \\
\hline 23 & $1,64 \mathrm{aB} A$ & $1,63 \mathrm{aA} A$ & $1,76 \mathrm{aA} A$ & $1,66 \mathrm{aB} A$ & $1,38 \mathrm{bB} B$ & $1,80 \mathrm{aA} A$ & $1,70 \mathrm{aB} A$ & $1,43 \mathrm{bB} B$ & $1,70 \mathrm{aA} A$ \\
\hline \multirow[t]{2}{*}{30} & $1,89 \mathrm{aA} A$ & $1,74 \mathrm{aA} A$ & $1,82 \mathrm{aA} A$ & $1,93 \mathrm{aA} A$ & $1,75 \mathrm{aA} A$ & $1,86 \mathrm{aA} A$ & $1,94 \mathrm{aA} A$ & $1,66 \mathrm{bA} A$ & $1,80 \mathrm{aA} A$ \\
\hline & \multicolumn{9}{|c|}{ Produção de grãos } \\
\hline 2 & $13,90 \mathrm{aA} A$ & $10,81 \mathrm{aA} A$ & $14,87 \mathrm{aA} B$ & $8,54 \mathrm{aB} A$ & $5,47 \mathrm{aA} A$ & $11,57 \mathrm{aA} B$ & $1,84 \mathrm{bB} B$ & $0,01 \mathrm{bB} B$ & $25,75 \mathrm{aA} A$ \\
\hline 9 & $16,25 \mathrm{aA} A$ & $5,10 \mathrm{bB} A$ & $18,70 \mathrm{aA} A$ & $7,39 \mathrm{bB} B$ & $0,02 \mathrm{cA} A$ & $16,16 \mathrm{aA} A$ & $2,89 \mathrm{bB} B$ & $0,02 \mathrm{bB} A$ & $17,28 \mathrm{aB} A$ \\
\hline 16 & $9,18 \mathrm{aB} A$ & $0,46 \mathrm{bB} A$ & $12,62 \mathrm{aA} B$ & $6,53 \mathrm{bB} A$ & $1,41 \mathrm{bA} A$ & $15,80 \mathrm{aA} B$ & $8,16 \mathrm{bB} A$ & $0,01 \mathrm{cB} A$ & $21,37 \mathrm{aA} A$ \\
\hline 23 & $8,46 \mathrm{aB} A$ & $1,84 \mathrm{bB} A$ & $11,09 \mathrm{aA} B$ & $8,83 \mathrm{aB} A$ & $2,02 \mathrm{bA} A$ & $13,17 \mathrm{aA} B$ & $6,31 \mathrm{bB} A$ & $1,37 \mathrm{bB} A$ & $17,67 \mathrm{aB} A$ \\
\hline 30 & $14,24 \mathrm{aA} B$ & $8,74 \mathrm{bAA}$ & $14,61 \mathrm{aA} B$ & $19,34 \mathrm{aA} A$ & $6,49 \mathrm{bA} A$ & $15,65 \mathrm{aA} B$ & $21,95 \mathrm{aA} A$ & $7,32 \mathrm{bA} A$ & $24,17 \mathrm{aA} A$ \\
\hline
\end{tabular}

${ }^{(1)}$ Médias seguidas de mesma letra, minúscula nas linhas e maiúscula nas colunas, dentro de cada tratamento inseticida e na testemunha, e mesma letra maiúscula em itálico nas linhas, para um mesmo fator de inoculação, entre os tratamentos inseticidas e a testemunha, não diferem entre si pelo teste de Scott-Knott, a 5\% de probabilidade. ${ }^{(2)}$ Idade das plantas (dias após a emergência) no momento em que foram submetidas às cigarrinhas.

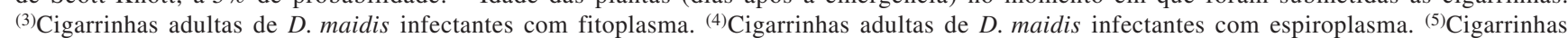
adultas de D. maidis sadias. 
milho causam menores danos à produção (Hruska \& Peralta, 1997; Massola Júnior et al., 1999).

No experimento em campo, a utilização dos inseticidas para controle da cigarrinha não resultou em redução significativa na incidência de enfezamentos e aumento da produção. A incidência de enfezamentos variou de $19 \%$ (sementes tratadas com imidacloprid e pulverização das plantas com thiamethoxan aos 10 DAE) a $36,4 \%$ (sementes não tratadas e pulverização das plantas com thiamethoxan aos 10 DAE). Observou-se que a produtividade variou de $3.347 \mathrm{~kg} \mathrm{ha}^{-1}$ (sementes não tratadas e pulverização das plantas com imidacloprid aos 10 DAE) a $4.631 \mathrm{~kg} \mathrm{ha}^{-1}$ (sementes não tratadas e pulverização das plantas com imidacloprid aos $10 \mathrm{e}$ 20 DAE). Considerando-se os resultados observados no experimento conduzido sob condições controladas, esse resultado obtido em campo não era esperado. Assim, é possível que a entrada contínua de cigarrinhas infectantes na área experimental durante o desenvolvimento da cultura tenha apresentado um efeito maior de disseminação dos fitopatógenos que a capacidade dos inseticidas de reduzirem o inóculo inicial de molicutes. A cigarrinha-do-milho tem sido apontada como uma espécie migratória, que permanece na cultura durante todo o ciclo, abandonando os campos de milho senescentes, e possivelmente, utilizando-se de correntes de vento para localização de novos campos para a colonização (Taylor et al., 1993; Oliveira, 2000). As cigarrinhas infectantes podem ter entrado na área do experimento, após o período residual do tratamento inseticida.

\section{Conclusões}

1. Em viveiro telado, o tratamento inseticida de sementes de milho com imidacloprid ou com thiamethoxan reduz a população inicial de cigarrinhas Dalbulus maidis e o inóculo inicial dos molicutes, particularmente quando as cigarrinhas se alimentam em plântulas até nove dias após a emergência.

2. Em condições de campo, o tratamento de sementes com os inseticidas testados não assegura a redução na incidência de enfezamentos.

3. O imidacloprid é mais eficaz que thiamethoxan, no controle de Dalbulus maidis por meio do tratamento de sementes.

\section{Agradecimentos}

Aos funcionários da Embrapa Milho e Sorgo, pelo auxílio na instalação e condução dos experimentos; ao CNPq, pela concessão de bolsa.

\section{Referências}

ABBOTT, W.S. A method of computing the effectiveness of on insecticide. Journal Economic Entomology, v.18, p.265-267, 1925.

BHIRUD, K.M.; PITRE, H.N. Bioactivity of systemic insecticides in corn - relationship to leafhopper Homoptera-Cicadellidae vector control and corn stunt disease incidence. Journal of Economic Entomology, v.65, p.1134-1140, 1972.

BOITEAU, G.; OSBORN, W.P.L.; DREW, M.E. Residual activity of imidacloprid controlling Colorado potato beetle (Coleoptera: Chrysomelidae) and three species of potato colonizing aphids (Homoptera: Aphidae). Journal of Economic Entomology, v.90, p.309-319, 1997.

BROADBENT, L. Insecticidal control of the spread of plant viruses. Annual Review of Entomology, v.2, p.339-354, 1957.

FERREIRA, D.F. Análises estatísticas por meio do Sisvar para Windows versão 4.0. In: REUNIÃO ANUAL DA REGIÃO BRASILEIRA DA SOCIEDADE INTERNACIONAL DE BIOMETRIA, 45., 2000, São Carlos. Anais. São Carlos: UFSCAR, 2000. p.255-258.

GUSSIE, J.S.; FLETCHER, J.; CLAYPOOL, P.L. Movement and multiplication of Spiroplasma kunkelii in corn. Phytopathology, v.85, p.1093-1098, 1995.

HRUSKA, A.J.; PERALTA, M.G. Maize response to corn leafhopper (Homoptera: Cicadellidae) infestation and achaparramiento disease. Journal of Economic Entomology, v.90, p.604-610, 1997.

LEGRAND, A.I.; POWER, A.G. Inoculation and acquisition of maize bushy stunt mycoplasma by its leafhopper vector Dalbulus maidis. Annals of Applied Biology, v.125, p.115-122, 1994.

MARKHAM, P.G.; ALIVIZATOS, A.S. The transmission of corn stunt spiroplasma by natural and experimental vectors. In: INTERNATIONAL MAIZE VIRUS DISEASE COLLOQUIUM AND WORKSHOP, 1982, Wooster. Proceedings. Wooster: The Ohio State University, Ohio Agricultural Research and Development Center, 1983. p.56-61. Editado por D.T. Gordon, J.K. Knoke, L.R. Nault, R.M. Ritter.

MASSOLA JÚNIOR, N.S.; BEDENDO, I.P.; AMORIM, L.; LOPES, J.R.S. Quantificação de danos causados pelo enfezamento vermelho e enfezamento pálido do milho em condições de campo. Fitopatologia Brasileira, v.24, p.136-142, 1999.

OLIVEIRA, C.M. de. Variação genética entre populações de Dalbulus maidis (DeLong \& Wolcott, 1923) (Hemiptera: Cicadellidae) e mecanismos de sobrevivência na entressafra do milho. 2000. 167p. Tese (Doutorado) - Escola Superior de Agricultura Luiz de Queiroz, Piracicaba. 
OLIVEIRA, C.M. de; LOPES, J.R.S. Técnicas para criação da cigarrinha-do-milho e inoculação de molicutes e vírus em planta. In: OLIVEIRA, E. de; OLIVEIRA, C.M. de (Ed.). Doenças em milho: molicutes, vírus, vetores e mancha por Phaeosphaeria. Brasília: Embrapa Informação Tecnológica, 2004. p.89-116.

OLIVEIRA, C.M. de; MOLINA, R.M.S.; ALBRES, R.S.; LOPES, J.R.S. Disseminação de molicutes do milho a longas distâncias por Dalbulus maidis (Hemiptera: Cicadellidae). Fitopatologia Brasileira, v.27, p.91-95, 2002a.

OLIVEIRA, E. de; CARVALHO, R.V.; DUARTE, A.P.; ANDRADE, R.A.; RESENDE, R.O.; OLIVEIRA, C.M. de; RECCO, P.C. Molicutes e vírus em milho na safrinha e na safra de verão. Revista Brasileira de Milho e Sorgo, v.1, p.38-46, 2002b.

OLIVEIRA, E. de; OLIVEIRA, C.M. de; MAGALHÃES, P.C.; ANDRADE, C.L.T.; HOGENHOUT, S.A. Spiroplasma and phytoplasma infection reduce kernel production, and nutrient and water contents of several but not all maize cultivars. Maydica, v.50, p.171-178, 2005.

OLIVEIRA, E. de; OLIVEIRA, C.M. de; SOUZA, I.R.P.; MAGALHÃES, P.C.; CRUZ, I. Enfezamentos em milho: expressão de sintomas foliares, detecção do molicutes e interações com genótipos. Revista Brasileira de Milho e Sorgo, v.1, p.53-62, 2002c.

OLIVEIRA, E. de; WAQUIL, J.M.; PINTO, N.F.J.A. Doenças causadas por fitopatógenos transmitidos por insetos: complexo enfezamento/mosaico. In: SEMINÁRIO SOBRE A CULTURA DO MILHO "SAFRINHA", 4., 1997, Assis. Anais. Assis: IAC/CDV, 1997. p.87-94.

PEDIGO, L.P. Conventional insecticides. In: PEDIGO, L.P. (Ed.). Entomology and pest management. New Jersey: Prentice Hall, 1999. p.373-431.

PERFECTO, I. Indirect and direct effects in a tropical agroecosystem: the maize-pest-ant system in Nicaragua. Ecology, v.71, p.2125-2134, 1990.
PIMENTEL-GOMES, F. Curso de estatística experimental. 14.ed. São Paulo: Nobel, 2000. 477p.

POWER, A.G. Influence of plant spacing and nitrogen-fertilization in maize on Dalbulus maidis (Homoptera: Cicadellidae), vector of corn stunt. Environmental Entomology, v.18, p.494-498, 1989.

POWER, A.G. Plant community diversity, herbivore movement, and an insect-transmitted disease of maize. Ecology, v.68, p.16581669, 1987.

RAMIRO, Z.A.; BATISTA FILHO, A.; CINTRA, E.R.R. Eficiência do inseticida Actara Mix $110+220$ ce (thiamethoxam + cipermetrina) no controle de percevejos-pragas da soja. Arquivos do Instituto Biológico, v.72, p.235-243, 2005.

SHURTLEFF, M.C. Compendium of corn diseases. $2^{\text {nd }}$ ed. St. Paul: American Phytopathological Society, 1980. 105p.

SILVA, R.G.; GALVÃO, J.C.C.; MIRANDA, G.V.; OLIVEIRA, E. de. Controle genético da resistência aos enfezamentos do milho. Pesquisa Agropecuária Brasileira, v.38, p.921-928, 2003.

SILVA, R.G.; GALVÃO, J.C.C.; MIRANDA, G.V.; OLIVEIRA, E. de. Identificação dos níveis e fontes de resistência aos enfezamentos do milho. Revista Brasileira de Milho e Sorgo, v.1, p.18-29, 2002.

TAYLOR, R.A.J.; NAULT, L.R.; STYER, W.E. Experimentalanalysis of flight activity of three Dalbulus leafhoppers (Homoptera: Auchenorrhyncha) in relation to migration. Annals of the Entomological Society of America, v.86, p.655-667, 1993.

TSAI, J.H.; STEINBERG, B.; FALK, B.W. Effectiveness and residual effects of seven insecticides on Dalbulus maidis (Homoptera: Cicadellidae) and Peregrinus maidis (Homoptera: Delphacidae). Journal of Entomological Science, v.25, p.106-111, 1990.

ZAGONEL, J.; REGHIN, M.Y.; DALLA PRIA, M.; KUNZ, R.P. Avaliação de inseticidas no controle de Myzus persicae (Sulz.) (Homoptera: Aphididae) na cultura da alface. Horticultura Brasileira, v.20, p.514-515, 2002. 